University of New Orleans

ScholarWorks@UNO

2009

\title{
Morphology, magnetic and dynamic properties of artificial structures assembled by acoustic vibrations
}

\author{
K B. Paul \\ University of New Orleans \\ L Malkinski \\ University of New Orleans
}

Follow this and additional works at: https://scholarworks.uno.edu/phys_facpubs

Part of the Physics Commons

\section{Recommended Citation}

J. Appl. Phys. 106, 063905 (2009)

This Article is brought to you for free and open access by the Department of Physics at ScholarWorks@UNO. It has been accepted for inclusion in Physics Faculty Publications by an authorized administrator of ScholarWorks@UNO.

For more information, please contact scholarworks@uno.edu. 


\title{
Morphology, magnetic and dynamic properties of artificial structures assembled by acoustic vibrations
}

\author{
K. B. Paul ${ }^{\text {a) }}$ and L. Malkinski \\ Advanced Materials Research Institute (AMRI), University of New Orleans, New Orleans, Louisiana 70148, \\ USA
}

(Received 27 May 2009; accepted 5 August 2009; published online 18 September 2009)

\begin{abstract}
Cobalt micromagnetic particles with an average size of $\approx 1.6 \mu \mathrm{m}$ and varying total mass of the powder were assembled on patterned media with perpendicular magnetization by acoustic vibrations onto designed shapes reflecting the primary material. The replicas were studied with scanning electron microscopy, vibrating sample magnetometry, and ferromagnetic resonance spectroscopy. Their properties were significantly influenced by the shape anisotropy induced through the parent molds. A tendency in the development of the physical characteristics of the replicas was observed as their geometrical parameters changed. (C) 2009 American Institute of Physics.
\end{abstract}

[doi:10.1063/1.3223317]

\section{INTRODUCTION}

The magnetic, structural, and magnetoelastic properties of particle assemblies are determined by their individual unit as well as by the spatial distribution of clusters and agglomerates. The forces between well-separated particles are dipolar (also called magnetostatic or demagnetizing), and they diminish with increasing distance as $\sim 1 / r^{3}$.

The Co particles used in this work are polydomain and not separated. Therefore they are able to cluster and aggregate. Accordingly, the interparticle interactions in a cluster are dipolar and attractive.

The intercluster interactions reflect on a larger scale the interparticle interactions. When the exchange coupling anisotropy of two particles dominates the demagnetizing and the magnetocrystalline anisotropies, the particles cluster. ${ }^{2}$ This is frequently unwanted on uncontrolled level. Still, the clustering might have a positive impact in some experiments as discussed further in this work.

Quantitatively, in terms of exchange lengths, if intracluster exchange coupling of the magnetic particles is dominant, then the cluster size is larger than the magnetostatic exchange, and the magnetocrystalline exchange lengths. ${ }^{3}$ The influence of the characteristic length scales and geometrical effects on the average anisotropy field, magnetic relaxation, and magnetic inhomogeneities is insufficiently studied. ${ }^{4-6}$

It is established in physics of particle magnetism that the size and shape of the basic constituent significantly influence the macroperformance of the fabricated material. ${ }^{4,5}$ For instance, decreasing the particle size leads to an increase in its saturation magnetization $\left(4 \pi M_{s}\right)$ and coercivity $H_{c}{ }^{6}$. The Gilbert damping parameter $\alpha$ is generally larger for microparticles (0.5-0.6) compared to $\alpha$ for nanoparticles (0.30.45 ) (see, e.g., Ref. 5, Fig. 6 therein). Considerable effect on $\alpha$ [and the absorbed microwave power (MWP)] plays the systematic decrease in the particle size. In nanoparticle systems the surface effect and some related phenomena are more notably expressed, ${ }^{7}$ e.g., excessive spins on the oxide

\footnotetext{
${ }^{a)}$ Electronic mail: dr_kpaul@hotmail.com.
}

layers. Assemblies of microparticles are considered high MWP-loss matter with $\alpha \approx 0.5$ and above, and linewidths $\Delta H \geq 2-3$ kOe. For comparison, the corresponding bulk crystalline materials have $\alpha \leq 0.1{ }^{8}$

The clusters on a surface can couple to produce various sizes and shapes. If the clusters are additionally subjected to some kind of mechanical force, it can interfere with the coupling process. In these experiments the particles are placed on a conventional substrate, set on a patterned medium with perpendicular magnetization component, e.g., amorphous Fe$\mathrm{CoS}$ ribbon. The substrate with the particles and the magnetic pattern are subjected to vertical vibrations and the magnetic clusters arrange on the designed pattern.

Microwaves in the $1-5 \mathrm{GHz}$ frequency range are mainly used in applications such as mobile phones, wireless local area network (LAN) systems, or bluetooth devices. A prominent technique to prevent electromagnetic interference between microwave radiation and electric devices (large scale integration (LSI) and central processing unit (CPU)) is the use of microwave absorption materials. The thickness of the device is inversely proportional to the resonant frequency and the magnetic permeability of the material [see, e.g., Ref. 9, Eq. (2) therein]. To tune the absorbers for various frequency ranges above $5 \mathrm{GHz}$, there have been consistent efforts to make the relevant materials thinner. In this respect, rubber or resin compounds are unsustainable due to the decrease in volume fraction of the magnetic phase. ${ }^{9}$ The presented method of particle assembly in this work may have the potential of producing good quality absorbers, provided that it is optimized with the best particle size, shape, and experimental conditions.

The main goal of this work is to demonstrate a method of organizing magnetic microparticles on vibrating patterned media with inherent perpendicular magnetization. The morphology and magnetic and dynamic properties of the artificial structures are studied, and conclusions are drawn for their practical applications as high-frequency absorbers. 


\section{EXPERIMENTAL DETAILS}

The microsize Co powders, with an average diameter of $1.6 \mu \mathrm{m}$, were purchased from Alfa Aesar chemicals and marked as $99.8 \%$ pure. The particles and replicas were analyzed with scanning electron microscopy (SEM) measurements to verify a mean particle size of $1.56 \mu \mathrm{m}$ with $\log$ normal distribution of the size and elongated shape with an aspect ratio of $\approx 3: 1$. The SEM images also elucidated the basic morphology of the assemblies.

The arrangement of the particles on designed areas was made with a low-voltage actuator PSt150/5/20 VS10, Piezosystem Jena, fed by a power supply ENV800. ${ }^{10}$ In these experiments, the actuator was attached perpendicular to the sample plane to initiate vertical vibrations with an amplitude of $A \approx 2 \mu \mathrm{m}$ and sweeping frequencies $f$ in the acoustic range. The substrate with the particles and the magnetic pattern are subjected to these vibrations. The related mechanical force $F$ is equal to $m A f^{2} \cos (2 \pi f t)$, where $m$ is the mass of a particle (or cluster), $t$ is the time of the vibration, and the rest of the parameters are as introduced. The energy, associated with this mechanical force, is imposed on the clustering particles. When commensurate with the intercluster exchange energy, it can prevent from further clustering by breaking the clusters into smaller ones. The system is set in the vertical magnetic field of the patterned medium. Consequently the clusters arrange on the designed pattern, and the inadequately large aggregations are tossed away from the substrate at the appropriate frequency of the vibration.

General magnetic characterization of the structures was achieved with a Lake Shore vibrating sample magnetometer. The basic characteristics of the assembled samplesaturation magnetization, reduced remanence, $M_{r} / M_{s}$, and coercivity-were estimated from measured hysteresis loops at room temperature, the measurements made in in-plane and out-of-plane orientations of the assembly, to test the influence of the particle assembling procedure.

To investigate for possible applications of these materials, ferromagnetic resonance (FMR) measurements were performed, and the Gilbert effective damping parameter was calculated. The room-temperature measurements were made with an $X$-band Bruker spectrometer EMX300. The sample was placed in the cavity in the parallel direction to the sweeping dc magnetic field $H$, and spectra were recorded at relevant angle steps $\left(10^{\circ}\right.$ or more). A resonance frequency wave ( $\mathrm{rfw}$ ) with an amplitude of $10 \mathrm{mOe}$ and frequency $f$ $\approx 10 \mathrm{GHz}$ was simultaneously introduced into the cavity. The magnetic spins, in precession in the increasing dc field at the frequency of the wave which is the resonance frequency, absorb the microwave power. The measured field derivative of the absorbed power $d P / d H$ versus $H$ is informative of important dynamic characteristics of the material discussed in Sec. III B.

\section{RESULTS AND DISCUSSION}

\section{A. Morphology and static magnetic measurements}

In Fig. 1 is displayed the field emission SEM (FESEM) image of a basic $3 \times 1 \mathrm{~mm}^{2}$ structure formed by Co particles covered with $100 \mathrm{~nm} \mathrm{Au}$ for these measurements. It is ob-

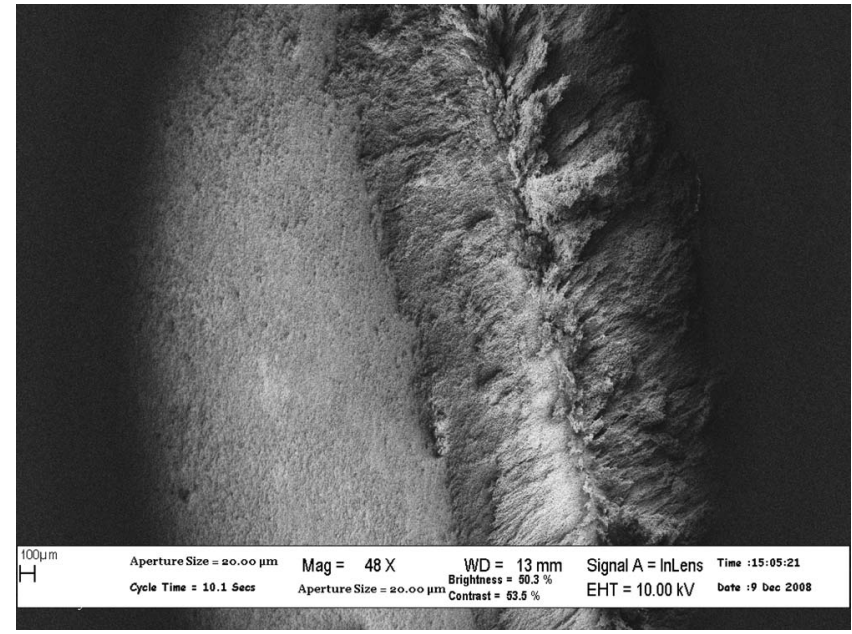

FIG. 1. FESEM image of a $3 \times 1 \mathrm{~mm}^{2}$ structure formed of Co particles before wrapping for macromagnetic measurements.

served that the powder structure consists of magnetic whiskers, some pointing outside the sample plane, well seen along the pole line of the parent material.

The macromagnetic state in these materials was assessed by the $M(H)$-plots measured at $300 \mathrm{~K}$ and the basic characteristics estimated from them. Two series of materials were studied: the first with widths of $1 \mathrm{~mm}$ and lengths varying from 1 to $6 \mathrm{~mm}$ and the second with widths of $1.3 \mathrm{~mm}$ and same lengths as in the first series of samples.

In the patterned medium the anisotropy depends on the parent material as well as the shape of the magnet. Consequently the magnetic properties can be engineered by the shape. The parent medium in these experiments has inherent perpendicular anisotropy; additionally, it is shaped as twofold symmetry rectangular magnets with millimeter size and decreasing aspect ratio as the length increases. The Co powder is arranged on the substrate by the acoustic vertical vibrations and it exhibits the planar shape of the parent material. The thicknesses are determined by the saturation mass of the complex entity: substrate and structure. They are usually $1.15 \pm 0.1 \mathrm{~mm}$ molded by the major forces acting on the particle system, attractive to the parent substrate and mechanical vibrations, which break and dissipate the large clusters and agglomerations.

Typical hysteretic measurements corresponding to the 3 mm long powder sample from the first series are shown in Fig. 2 for parallel (par) and perpendicular (per) positions of the sample plane to the applied field. The plots are characterized with a steep increase in the magnetization at low fields followed by a linear saturating behavior. In the inset of Fig. 2 is shown the low-field hysteretic behavior of the 1 $\times 3 \mathrm{~mm}^{2}$ sample for the in-plane orientation. It is seen that the hysteresis loop is double jump, characteristic for incoherent switching. ${ }^{11}$ Similar incoherent switching was observed for all samples from the first series when the magnetic field was applied parallel to the sample plane. In the perpendicular alignment of the field, the switching is coherent. The modification of the switching mode is obviously due to the geometrical aspects of the patterned medium; also it is influenced by the sole properties of fabricated structures. ${ }^{12}$ In the 


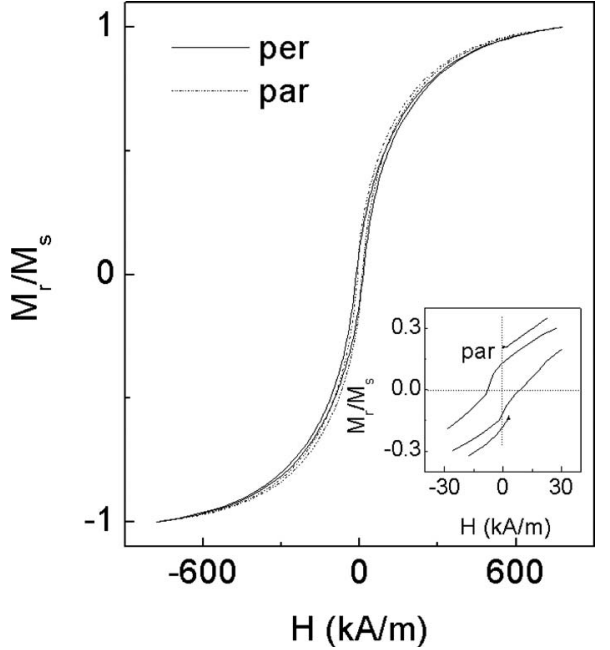

FIG. 2. Typical $M-H$ plots at room temperature for the $1 \times 3 \mathrm{~mm}^{2}$ sample in perpendicular (per) and parallel (par) orientations to the field. In the inse is displayed a detailed $M(H)$ view at the low-field range for the parallel orientation.

parent material, there is a pole area which results in nonhomogeneous distribution of the particles on the plane. Although a general principle of minimizing the surface area of the pole faces is valid, the nonhomogeneity affects the results especially in longer samples.

It is a general observation, seen from $M(H)$-plots and the results summarized in Table I, that these materials have lowcoercive fields, low remanence, and low squareness of the hysteresis loops. The coercivities are higher in the out-ofplane direction for all samples, and the magnetic remanence is higher in the in-plane direction (approximately twice higher than the out-of-plane remanence). Therefore, the fabricated structures exhibit perpendicular magnetic anisotropy and near-in-plane isotropy similar to the parent material.

The changes in the physical properties of these structures are a direct result of varying their size. To quantify the effect of the shape anisotropy, the coercivity was measured from the hysteresis loops, and the results are displayed in Fig. 3 as $H_{c}$ versus sample length $L$. The plot with the dark circles represents the results for the perpendicular magnetization of the narrow structures, and the open circles - the in-plane results.

The designed magnetic materials have demagnetizing fields, which scale as $t / a$, with $t$ the thickness of the magnet and $a$ its size $=\sqrt{ }$ area. An interplay between the induced

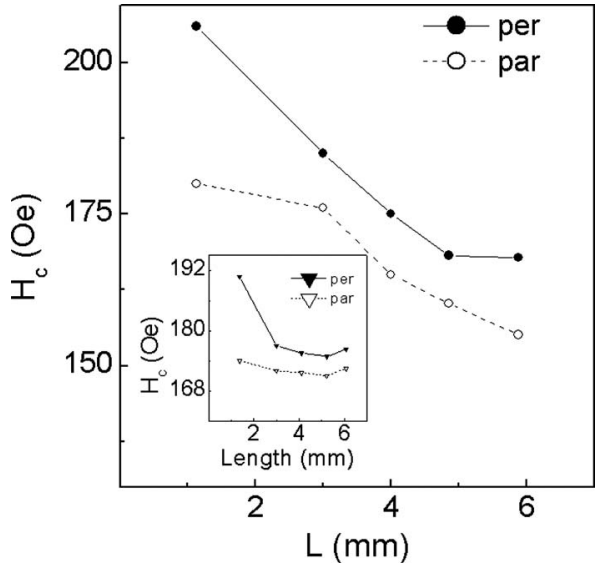

FIG. 3. $H_{c}$ vs $L$ for the structures with a width of $1 \mathrm{~mm}$; the lines serve to guide the eyes. The results for the structures with a width of $1.3 \mathrm{~mm}$ are presented in the inset of the figure.

shape anisotropy and the structure's demagnetizing field may lead to coercivities which increase when the size of the magnet decreases: an effect well described for nanomagnets with a constant aspect ratio of $1: 2$ in Ref. 12 [see Fig. 3(a) therein].

The fabricated structures in this work are rectangular with changing aspect ratio. When the aspect ratio decreases (length increases), the macromagnetic parameters may increase when the change in the demagnetizing field lags the change in shape anisotropy caused by an increase in length. The total morphology and magnetic behavior can be affected as a result.

\section{B. Dynamic magnetic properties}

The FMR and the measured field derivative of the absorbed MWP $d P(H) / d H$ inform about the damping in the material and possibility for the presence of magnetic inhomogeneities in it. The results place emphasis on the positions of the FMR lines, their widths, and shapes. The positions are informative about the resonant dc field $\left(H_{\text {res }}\right)$ at the employed frequency. The linewidths $(\Delta H)$ and the shapes elucidate damping, associated relaxation processes, and inhomogeneities.

The absorption spectral lines in these experiments are obtained from the integration of the measured $d P(H) / d H$ profiles at $f \approx 10 \mathrm{GHz}$ and room temperature after baseline corrections. It is possible to calculate the linewidth as the

TABLE I. Parameters of some fabricated materials. Per denotes out-of-plane parameters and par denotes in-plane parameters. Par1 and par2 denote parameters associated with two in-plane directions.

\begin{tabular}{|c|c|c|c|c|c|c|c|c|c|c|}
\hline \multirow{3}{*}{$\begin{array}{l}\text { Length } \\
(\mathrm{mm})\end{array}$} & \multicolumn{6}{|c|}{ First series (width of $1 \mathrm{~mm}$ ) } & \multicolumn{4}{|c|}{ Second series (width of $1.3 \mathrm{~mm}$ ) } \\
\hline & \multicolumn{2}{|c|}{ Per } & \multicolumn{2}{|c|}{ Par1 } & \multicolumn{2}{|c|}{ Par2 } & \multicolumn{2}{|c|}{ Per } & \multicolumn{2}{|c|}{ Par } \\
\hline & $H_{c}$ & $M_{r} / M_{s}$ & $H_{c}$ & $M_{r} / M_{s}$ & $H_{c}$ & $M_{r} / M_{s}$ & $H_{c}$ & $M_{r} / M_{s}$ & $H_{c}$ & $M_{r} / M_{s}$ \\
\hline 1 & 206 & 0.070 & 180 & 0.110 & 181 & 0.110 & 180 & 0.044 & 174 & 0.117 \\
\hline 3 & 185 & 0.060 & 176 & 0.115 & 176 & 0.120 & 177 & 0.060 & 172 & 0.126 \\
\hline 4 & 175 & 0.053 & 165 & 0.123 & 166 & 0.120 & 188 & 0.048 & 175 & 0.120 \\
\hline 5 & 177 & 0.055 & 170 & 0.115 & 171 & 0.113 & 184 & 0.060 & 172 & 0.120 \\
\hline 6 & 169 & 0.087 & 172 & 0.120 & 172 & 0.120 & 178 & 0.056 & 174 & 0.120 \\
\hline
\end{tabular}




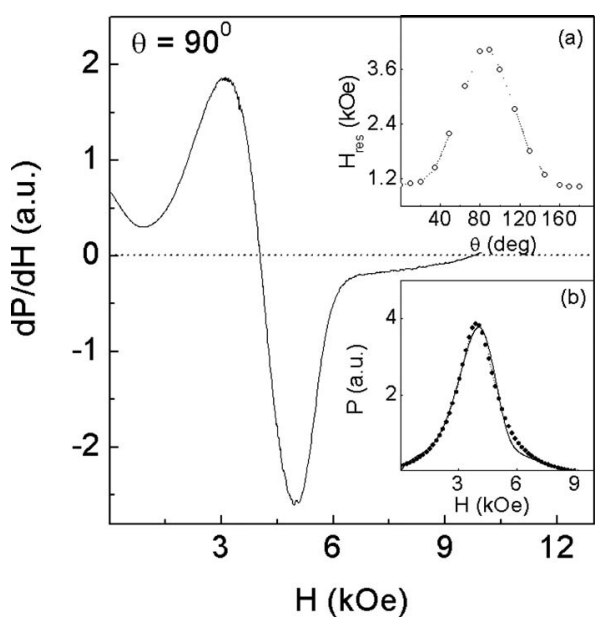

FIG. 4. $d P / d H$ vs $H$ measured with the sample plane perpendicular to the field. In inset (a) is presented the angular dependence of the resonance field $H_{\text {res }}$. In inset (b) is introduced the integrated $P(H)$ line; the dots represent the Lorentzian fit to $P(H)$.

peak to peak values of the experimental $d P(H) / d H$ versus $H$-plots. ${ }^{5,6,13}$ As discussed in Ref. 6 and through the experiences herein, the second method may produce higher values of $\Delta H$ at frequencies below $20 \mathrm{GHz}$. In the discussion further in the work are used results from the first method of calculation.

In Fig. 4 is presented a FMR measurement for the 1 $\times 1 \mathrm{~mm}^{2}$ sample from the first series made in the perpendicular plane direction to the field. In inset (a) of Fig. 4 is presented the azimuthal angular dependence of the resonance field $H_{\text {res }}(\theta)$. In inset (b) of the figure is shown the integrated $P(H)$ plot; the dot line with the symbols is the Lorentzian graph fitted to the measured and integrated $P(H)$.

As clearly observed in inset (a), $H_{\text {res }}$ is the highest at $90^{\circ}$, i.e., when the sample plane is perpendicular to the sweeping field. This dependence is analogous to $H_{\text {res }}(\theta)$ for flat polycrystalline soft samples and nanocomposites with shape anisotropy. ${ }^{13}$

The resonant fields in inset (a) are associated with the maxima in the integrated absorption profiles. ${ }^{6}$ They are related to the uniform-mode angular frequencies as $\omega_{\text {res }}$ $=\gamma\left(H_{\text {res }}+H_{\text {int }}\right){ }^{5,6,13}$ The parameter $\gamma$ is the effective gyromagnetic ratio and $H_{\text {int }}$ is an average internal field, reflecting the effect of the local inhomogeneities in the material.

The gyromagnetic ratio $\gamma$ can be determined experimentally from measurements at several resonant frequencies $\omega_{\text {res }} / 2 \pi$. Another approach to determine $H_{\text {int }}$ is to use available data in the literature for $\gamma$ (or the spectroscopic splitting factor $g$ since $\gamma=g \mu_{B} / \hbar ; \mu_{B}$ is the Bohr magneton and $\hbar$ is the reduced Planck constant). The second method is used when the experiments are performed at one frequency only, ${ }^{13}$ as in the presented work.

The close-to-Lorentzian shape integrated $P(H)$ absorption spectra are generally distorted for these structures; they are slightly asymmetric to the maximum, as observed, for instance, in Fig. 4 inset (b) for the sample with $1 \times 1 \mathrm{~mm}^{2}$ dimensions. Similar to the presented results asymmetry and non-Lorentzian structure of the peak-absorption line are discussed for $1 \mu \mathrm{m} \quad \gamma-\mathrm{Fe}_{2} \mathrm{O}_{3}$ particle compounds. ${ }^{5}$ The distor-

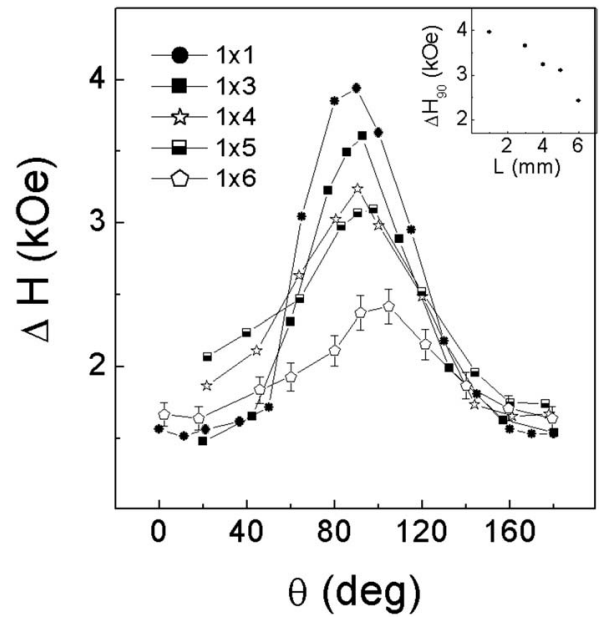

FIG. 5. Angular dependence of the estimated linewidths for the first series of samples (widths of $1 \mathrm{~mm}$ and lengths varying from 1 to $6 \mathrm{~mm}$ ). The data for sample $1 \times 6$ are presented with $5 \%$ error bars. In the inset is introduced $\Delta H_{90}(L)$. The lines in the figure are guides for the eyes.

tions are explained with nonuniform magnetization motions due to the orientation distribution of the local anisotropy fields. ${ }^{5}$ This seems to be a probable cause for the line distortions of the artificial structures studied in this work.

The related samples have shape anisotropy, and the Co particles in them are polycrystalline with no preferred orientation. Therefore, the edgy current losses are possibly nonnegligible and they may also contribute to the distortion in the shape of $P(H){ }^{14}$

The linewidth $\Delta H$ is the measure of the rate at which the energy is transferred from the precessing magnetic moments of the sample to its lattice. A rapid transfer results in a strong damping of the precessional motion, and accordingly short relaxation times, which broadens the spectral line overall. Effectively, $\Delta H$ is defined as the full width at half maximum of $P(H)$. Two main contributions to the linewidth are determined in an in-depth analysis in Ref. 5: intrinsic and extrinsic. While the intrinsic part is a fundamental characteristic of the particular material, the origins of the extrinsic constituent are mostly related to the sample fabrication procedure. The latter may affect the inhomogeneities and therefore the internal static magnetic fields, also the anisotropic dispersion within the sample. The inhomogeneous component of $\Delta H$ is frequency independent and the homogeneous part depends on $\omega_{\text {res }} / 2 \pi .^{5}$

In Fig. 5 is displayed the dependence of the linewidth on the angle enclosed between the sample plane and the magnetic field for the first series of samples with widths of $1 \mathrm{~mm}$. In the inset of Fig. 5 is presented the dependence of the linewidth at the sample plane perpendicular to the magnetic field $\left(\Delta H_{90}\right)$ on the sample length $\Delta H_{90}(L)$. The angular anisotropy in $\Delta H$ is best observed with the shortest sample $\left(1 \times 1 \mathrm{~mm}^{2}\right)$, and it can increase to an additional $\approx 2 \mathrm{kOe}$ as the structure is rotated from $0^{\circ}$ to $90^{\circ}$. The change in $\Delta H(\theta)$ is less pronounced when the sample length is increased, as seen in Fig. 5 and in its inset. Similar anisotropy in $\Delta H(\theta)$ was monitored for the second series of samples with widths of $1.3 \mathrm{~mm}$. The range of change in $\Delta H$ for the samples from the second series is between 4.2 and $5.8 \mathrm{kOe}$ and is best 
monitored in the shortest sample $(1 \times 1.3)$. It is smaller than the range of change in $\Delta H(\theta)$ for the first series-from 4.4 to $6.7 \mathrm{kOe}$. Thus, the anisotropy in $\Delta H$ is suppressed, or rather influenced by the demagnetizing fields, as the lengths of the materials are increased (see, e.g., Ref. 6, Appendix B for similar results). If an experimental error of determining the magnetic fields of $2 \%$ is assumed with the Bruker measuring equipment for FMR, and an error in calculations of $\Delta H$ $\approx 3 \%$ is taken up, the total systematic error of the estimated $\Delta H$ will not exceed $5 \%$ of the real $\Delta H$-values.

When the values of $\Delta H$ are of the same order as the resonance fields $H_{\text {res }}$, the analysis of the particular constituents in the relaxation process would require a more complex formalism than a single Lorentzian line, ${ }^{15}$ which is outside the scope of this work. Two-maximum absorption curves are predicted to explain the FMR results for suspensions of ferromagnetic particles in a theoretical work. ${ }^{16}$ There is a possibility of existing weaker modes associated with polydispersity of the particles. These weaker modes have frequencies close to the frequency of the dominant mode and may significantly distort its shape. ${ }^{5,17}$

The introduced physical characteristics $\Delta H, \alpha, \omega_{\text {res }}$, and $\gamma$ are related to describe the rfw damping in materials according to the relation $\alpha=1 / 2(\Delta H \cdot \gamma) / \omega_{\text {res. }}{ }^{6,13}$ The calculations based on the presented results reveal angular dependence (anisotropy) of the Gilbert damping parameter, with values of $\alpha$ ranging from 0.58 to $0.89( \pm 0.03)$ for the first series of these materials, in parallel and perpendicular directions to the field correspondingly. For the second series of materials with widths of $1.3 \mathrm{~mm}$, the values of $\alpha$ range from 0.61 to $0.80 \pm 0.04$ for parallel and perpendicular orientations of the sample plane, respectively. Also, there is an apparent dependence of $\alpha$ on the geometrical attributes of the sample, which can certainly be optimized for best performance of a device. Close Gilbert damping parameters are reported in Ref. 5 for microsize particle composites.

\section{CONCLUSIONS}

This work reports on a new method of particle assembly and formation of particle structures. It investigates the possibility to use these structures as high-frequency absorption and tuning materials. It is established that these structures can absorb MWP significantly with $\alpha \approx 0.6$ at $10 \mathrm{GHz}$ when placed in parallel to the magnetic field. Regarding the existing proportional relation of $\omega_{\text {res }}$ and $H_{\text {res }}$, it is expected that these materials can be applied successfully at higher frequencies to prevent electromagnetic interference in highfrequency devices. This statement is accompanied with the requirement to optimize these structures in terms of particle size and shape, packing density of the powder, and the specific material.

\section{ACKNOWLEDGMENTS}

The authors acknowledge the financial support from the National Science Foundation under NIRT Grant No. CCF0403673.

${ }^{1}$ P. Politi and M. G. Pini, Phys. Rev. B 66, 214414 (2002).

${ }^{2}$ G. Herzer, IEEE Trans. Magn. 26, 1397 (1990); in Handbook of Magnetic Materials, edited by K. H. J. Buschow (North-Holland, Amsterdam, 1997), Vol. 10.

${ }^{3}$ The magnetoelastic exchange length (MEL) is equal to $\sqrt{ } 2 A / \mu_{0} M_{s}{ }^{2}$. The magnetocrystalline exchange length (MCL) is equal to $\sqrt{ } A / K$. The saturation magnetization is represented by $M_{s}$, the crystalline anisotropy constant by $K$, and the exchange stiffness parameter is $A$. For Co in the bulk state, MEL and MCL are $3.30 \mathrm{~nm}$ and $0.55 \mathrm{~nm}$, correspondingly, with values for $A=13 \times 10^{-12} \mathrm{~J} / \mathrm{m}, \quad M_{s}=1.4 \times 10^{6} \mathrm{~A} / \mathrm{m}, \quad$ and $K=4.5$ $\times 10^{5} \mathrm{~J} / \mathrm{m}^{3}$

${ }^{4}$ C. Brosseau, J. Ben Youssef, P. Talbot, and A. M. Konn, J. Appl. Phys. 93, 9243 (2003)

${ }^{5}$ J. Ben Youssef and C. Brosseau, Phys. Rev. B 74, 214413 (2006).

${ }^{6}$ V. Castel, J. Ben Youssef, and C. Brosseau, J. Nanomater. 2007, 1 (2007).

${ }^{7}$ F. Bódker, S. Mórup, and S. Linderoth, Phys. Rev. Lett. 72, 282 (1994).

${ }^{8}$ P. W. Brouwer, Phys. Rev. B 58, R10135 (1998); Z. Celinski and B. Heinrich, J. Appl. Phys. 70, 5935 (1991).

${ }^{9}$ T. Maeda, S. Sugimoto, T. Kagotani, N. Tezuka, and K. Inomata, J. Magn. Magn. Mater. 281, 195 (2004).

${ }^{10} \mathrm{http}: / / \mathrm{www} \cdot$ piezojena.com/

${ }^{11}$ L. Torres, E. Martinez, L. Lopez-Diaz, and J. Iniguez, J. Appl. Phys. 89, 7585 (2001)

${ }^{12}$ R. P. Cowburn, D. K. Koltsov, A. O. Adeyeye, and M. E. Welland, Europhys. Lett. 48, 221 (1999).

${ }^{13}$ M. Parvadi-Horvath and L. J. Swartzendruber, IEEE Trans. Magn. 35, 3502 (1999).

${ }^{14}$ R. Rampsarad, P. Zurcher, M. Petras, M. Miller, and P. Renaud, J. Appl. Phys. 96, 519 (2004).

${ }^{15}$ J. H. van Vleck and V. F. Weisskopf, Rev. Mod. Phys. 17, 227 (1945).

${ }^{16}$ Y. L. Raikher and V. I. Stepanov, Phys. Rev. B 50, 6250 (1994).

${ }^{17} \mathrm{G}$. Viau, F. Ravel, O. Acher, F. Fievet-Vincent, and F. Fievet, J. Magn. Magn. Mater. 140-144, 377 (1995); P. Toneguzzo, O. Acher, G. Viau, F. Guillet, E. Bruneton, F. Fievet-Vincent, and F. Fievet, J. Appl. Phys. 81, 5546 (1997) 